\title{
Course of auditory vocal hallucinations in childhood: 5-year follow-up study
}

\author{
Agna A. Bartels-Velthuis, Gerard van de Willige, Jack A. Jenner, Jim van Os and Durk Wiersma
}

\section{Background}

In a baseline study among 7- and 8-year-old children with auditory vocal hallucinations, only limited functional impact was observed.

\section{Aims}

To assess 5-year course and predictors of auditory vocal hallucinations, as well as 5-year incidence and its risk factors.

\section{Method}

A sample of 337 children, 12 and 13 years of age, were reassessed on auditory vocal hallucinations and associated symptoms after a mean follow-up period of 5.1 years.

\section{Results}

The 5-year persistence and incidence rates were $24 \%$ and $9 \%$ respectively, with more new cases arising in urban areas. Both persistent and incident auditory vocal hallucinations were associated with problem behaviour in the clinical range of psychopathology as measured with the Child Behavior Checklist, particularly at follow-up, as well as with other psychotic symptoms, particularly at baseline. Persistence was predicted by baseline auditory vocal hallucinations severity, particularly in terms of external attribution of voices and hearing multiple voices, and was associated with worse primary school test scores and lower secondary school level.

\section{Conclusions}

First onset of auditory vocal hallucinations in middle childhood is not uncommon and is associated with psychopathological and behavioural comorbidity. Similarly, persistence of auditory vocal hallucinations in childhood is not uncommon and is associated with psychopathological, behavioural and cognitive alterations.

\section{Declaration of interest}

None.
Subclinical psychotic experiences in the general population are prevalent in both children and adults, and not generally associated with persistence over time or onset of psychiatric disorder. Nevertheless, literature reviews suggest that a small number will make the transition to a clinical psychotic disorder. ${ }^{1,2}$

In a previous study on a representative sample of 7- and 8-yearold children in The Netherlands, the prevalence of auditory vocal hallucinations was $9 \% .^{3}$ Although auditory vocal hallucinations in these children were mostly of limited functional impact, a subgroup with serious suffering was considered at risk for more severe psychotic outcomes.

The current study presents a 5-year follow-up of this sample (now 12 and 13 years of age) and examines the persistence and new incidence of auditory vocal hallucinations, as well as their clinical relevance in terms of problem behaviour, severity and associations with aetiological variables. In view of the baseline results and previous work in this area, it was hypothesised that:

(1) severity of auditory vocal hallucinations at baseline would predict persistence; ${ }^{4-6}$

(2) auditory vocal hallucinations would show associations with behavioural problems ${ }^{7-9}$ and other psychotic symptoms; ${ }^{10}$

(3) incidence and severity of auditory vocal hallucinations would be associated with environmental factors such as cannabis use $^{11-13}$ and urbanicity; ${ }^{14-16}$

(4) baseline characteristics of auditory vocal hallucinations suggesting external attribution and higher level of intrusion would be predictive of persistence; ${ }^{4-6}$

(5) auditory vocal hallucinations would be associated negatively with cognitive ability. ${ }^{17-20}$

\section{Method}

\section{Procedure}

From the case-control sample of the first wave ( $n=694$, of which 347 children with auditory vocal hallucinations), parents of 605 children ( $87 \%$, of which $50 \%$ with auditory vocal hallucinations) gave informed consent for follow-up. These parents were sent a notification letter by mail. Non-responders were sent a reminder followed by a second letter, if necessary. In case of persisting non-response, parents were contacted by telephone if their numbers could be traced.

Seven female interviewers (six students (bachelor degree) from the Department of Orthopedagogy and one psychology graduate, all from the University of Groningen) received extensive training by A.A.B.-V., G.v.d.W. and J.A.J. in conducting the interviews. First, they were introduced to the topic of auditory hallucinations, and then, with consent, observed several therapeutic sessions of patients receiving treatment at the Voices Outpatient Department of the University Medical Center Groningen. Interviewers were informed in detail about the structure and results of the baseline study and received training in the administration of instruments and in conducting interviews with children. Finally, they interviewed patients of the Voices Outpatient Department, under the supervision of G.v.d.W. and J.A.J. In addition to the formal interview training, several booster sessions were arranged to discuss interview and scoring procedures and to prevent interviewer 'drift'. Interviewers were instructed in a detailed protocol on how to approach families and how to conduct the interviews. In order to prevent bias, interviewers were unaware of the children's auditory vocal hallucination status at baseline. Children were interviewed at home, separately from their parents.

First, children were screened on the experience of hearing voices in the past 5 years; children with auditory vocal 
hallucinations were subsequently interviewed with the Auditory Vocal Hallucination Rating Scale (AVHRS). ${ }^{21}$ Given evidence for associations between auditory hallucinations and the use of substances ${ }^{11,22}$ and alterations in performance at school, ${ }^{17}$ information was also gathered in these domains. Parents completed the Child Behavior Checklist (CBCL), ${ }^{23}$ designed for children 4-18 years of age, and a sociodemographic questionnaire. Finally, parents were instructed on how to contact the research team in case they or their children had questions or worries resulting from the interview.

\section{Participants}

A total of 337 children were interviewed at follow-up, representing $49 \%$ of the baseline sample of 694 and $56 \%$ of the 605 that had consented to follow-up. Parents of 48 children were willing to complete the CBCL without their children being interviewed, 112 parents withdrew their informed consent and 108 families could not be contacted because of non-response or (re-)emigration.

Children at follow-up, similar to baseline, continued to be evenly distributed regarding baseline case or control status: 170 children with auditory vocal hallucinations (cases) and 167 children without auditory vocal hallucinations (controls). From the combined baseline and follow-up data, four groups could be distinguished:

1 children hearing voices at baseline and during the 5-year follow-up period: the persistent group;

2 children hearing voices at baseline but not during the 5-year follow-up period: the remitted group;

3 children not hearing voices at baseline but positive for auditory vocal hallucinations over the follow-up period: the incident group;

4 children not hearing voices at both baseline and follow-up: the referent group.

The persistent group was defined as children hearing voices in the year before the baseline interview and (at least occasionally) during one other year over the follow-up period. The incident group was defined as children who heard voices for the first time after baseline assessment, with a minimum duration of 3 months.

\section{Instruments}

\section{Children}

The 16-item AVHRS is a structured interview, ${ }^{21}$ with the characteristics of hearing voices (e.g. frequency, attribution, duration, loudness, negative content, distress, anxiety, control, interference with thinking and with daily life) rated on a 5-point scale. Psychometric properties of the AVHRS are good. ${ }^{24}$ During booster sessions, DVD-recorded AVHRS interviews (of consenting patients attending the Voices Outpatient Department) were rated by all interviewers and A.A.B.-V. Total interrater agreement score (weighted Cohen's kappa) was 0.88 .

Use of substances (cannabis, synthetic drugs such as ecstasy, amphetamines, cocaine and heroin) was assessed by a self-report questionnaire, in order to avoid socially desirable answers.

\section{Cognitive ability}

In order to rate children's performance at school, children and parents were asked to provide the results of the national Dutch end-of-primary-school test, which assesses the continuation level for secondary education. The standard scores range from 1 to 50; children scoring 1-28 are qualified for vocational secondary education, and those scoring 29-50 for pre-academic secondary education. In addition, current secondary school level was assessed.

\section{Parents}

The CBCL/4-18 is a self-report questionnaire for parents regarding their child's behaviour. ${ }^{23}$ It consists of 113 items, grouped into nine syndrome scales of behavioural, social and physical functioning. Items are rated on a 3 -point scale $(0=$ not true; 1 = somewhat or sometimes true; $2=$ very true or often true). Parents also provided demographic data including their educational level and family income.

\section{Analysis}

Conform baseline analysis, ${ }^{3}$ an AVHRS severity index, was computed by recoding items to ' 0 ' = 'none or mild consequences' versus ' 1 ' = 'considerable to severe consequences'. Based on this index, two groups were defined: a severe auditory vocal hallucinations group (children scoring $\geqslant 5$ ) and a mild auditory vocal hallucinations group (scores $0-4$ ), that were contrasted with the referent group.

Degree of urbanisation was defined by the family's home address and split (conform baseline analysis ${ }^{3}$ ) into rural (somewhat urban and rural: ' 0 ') and urban (very, strongly and moderately urban: ' 1 '), based on data from the official classification of urbanicity provided by Statistics Netherlands (www.cbs.nl/en-GB/menu/home/default.htm?Languageswitch=on).

From the CBCL total score, the borderline clinical range of psychopathology was determined according to Achenbach ${ }^{23}$ (i.e. a cut-off score of 31 for both genders).

In order to avoid multiple testing, only CBCL main indices (internalising, externalising, total score and clinical range) were used. In addition, the 'thought problems' scale was used, as the items of this scale represent psychotic experiences and behaviour ('Strange behaviour', 'Strange ideas', 'Hears sounds or voices that aren't there', 'Sees things that aren't there', 'Stares blankly', 'Can't get his/her mind off certain thoughts', 'Repeats certain acts over and over'). Given that the item 'hearing things' overlaps with the dependent variable of auditory vocal hallucinations, analyses with the 'thought problems' scale were also carried out without this item, as well as with a more narrow selection representing the three (non-auditory vocal hallucinations) psychotic experiences: 'Strange behaviour', 'Strange ideas' and 'Sees things that aren't there'.

Secondary education was defined at two levels: low $(1=$ vocational $)$ and high $(0=$ pre-academic $)$. Socioeconomic status (SES) was derived from parental averaged educational levels and family income, resulting in three levels: low (score $=1)$, middle $($ score $=2)$ and high $($ score $=3)$.

Analyses were carried out using SPSS for Windows, version 16.0. Standard multinomial logistic regression, yielding odds ratios (OR) and 95\% confidence intervals (CI), were used to compare the four groups of children (incident, persistent, remitted and referent), with the referent group as the reference category. Significance tests were two-tailed with alpha set at 0.05 .

Differential non-response due to differences in help-seeking and psychiatric service use was tested by tracing the children anonymously at a group level through the Psychiatric Case Register North-Netherlands (PCR-NN) over the study period.

\section{Results}

\section{Sample characteristics, and incidence and prevalence rates of auditory vocal hallucinations}

Mean age of the follow-up sample was 13.1 years (s.d. $=0.5$, range 12.0-14.6). Mean interval between baseline and follow-up assessment was 5.1 years (s.d. $=0.4$ ). Attrition analyses (based on 
$n=694)$ showed that the participation rate was equal for baseline cases (49\%) and controls (48\%). Registration rates at the PCR-NN were not different for participating and non-participating children ( $8 \%$ v. $10 \%$; OR $=0.83,95 \%$ CI $0.49-1.40, P=0.48)$, and these groups did not differ in mean number of psychiatric service contacts: 2.5 (s.d. $=1.3$ ) in the participating children and 2.4 (s.d. =1.6) in the non-participating children $(\mathrm{OR}=1.06,95 \% \mathrm{CI}$ $0.75-1.51, P=0.73)$. The participation rate for girls was significantly higher than for boys $\left(53 \%\right.$ v. $44 \% ; \chi^{2}=5.50$, $P=0.02$ ). There was no evidence for differential attrition as a function of age and urbanicity. A flow chart of follow-up participants $(n=337)$ is presented in Fig. 1.

Most children in the follow-up sample (90\%) lived in a rural environment. Socioeconomic status was evenly distributed $(31 \%$ low, 39\% middle and 30\% high). Mean score on the end-ofprimary-school test was 37.2 (s.d. $=8.7$, range $9-50$ ); $53 \%$ of the children were attending higher-level secondary education. In Table 1, demographic data by follow-up status are presented.

\section{Course of auditory vocal hallucinations and predictors of persistence}

The persistence rate of auditory vocal hallucinations was $23.5 \%$ and the 5-year cumulative incidence rate in the baseline control sample was $9.0 \%$. Mean duration of hearing voices in the persistent group was 5.7 years $($ s.d. $=1.9)$ and in the incident group 3.5 years $($ s.d. $=1.7)$.

Children still hearing voices after 5 years, compared with children in the remitted group, at baseline more often belonged to the group with severe auditory vocal hallucinations (40\% $\mathrm{v}$. $21 \%, \mathrm{OR}=2.54,95 \%$ CI 1.19-5.45, $P=0.016$ ) (Table 2). Examining distinct baseline auditory vocal hallucination characteristics revealed that hearing more than one voice discriminated best between persistence and remission of baseline auditory vocal hallucinations $(\mathrm{OR}=2.81,95 \% \mathrm{CI} 1.09-7.23, P=0.032)$. Also, attribution of voices to an external source (e.g. deceased family members or an extra-terrestrial source) discriminated between persistence and remission $(\mathrm{OR}=2.35,95 \%$ CI 1.11-4.97, $P=0.025)$.

\section{Auditory vocal hallucinations and cognitive ability}

Children with persistent auditory vocal hallucinations, compared with the referent group, displayed lower end-of-school cognitive test scores (mean score 32 v. 37; OR $=1.07,95 \%$ CI 1.02-1.12, $P=0.004)$ and more often had lower secondary school education $(71 \%$ v. $48 \%$; OR $=2.63,95 \%$ CI $1.21-5.68, P=0.014)$. The incident group, however, did not differ from the referent group. Children in the remitted group scored highest on both cognitive ability outcome measures (Table 2).

\section{Problem behaviour}

Compared with the referent group, children with persistent auditory vocal hallucinations, as well as children with incident auditory vocal hallucinations, displayed higher scores on the 'thought problems' scale of the CBCL at both baseline and follow-up (Table 3). Also, when the item on auditory hallucinations (item 40) was excluded from this scale, these differences remained ('thought problems' baseline: persistent auditory vocal hallucinations, $\mathrm{OR}=1.53,95 \%$ CI $1.07-2.19$, $P=0.02$; incident auditory vocal hallucinations, $\mathrm{OR}=1.82,95 \%$ CI 1.10-3.02, $P=0.02$; 'thought problems' follow-up: persistent auditory vocal hallucinations, $\mathrm{OR}=1.27,95 \%$ CI $0.93-1.75$, $P=0.14$; incident auditory vocal hallucinations, $\mathrm{OR}=1.82,95 \%$

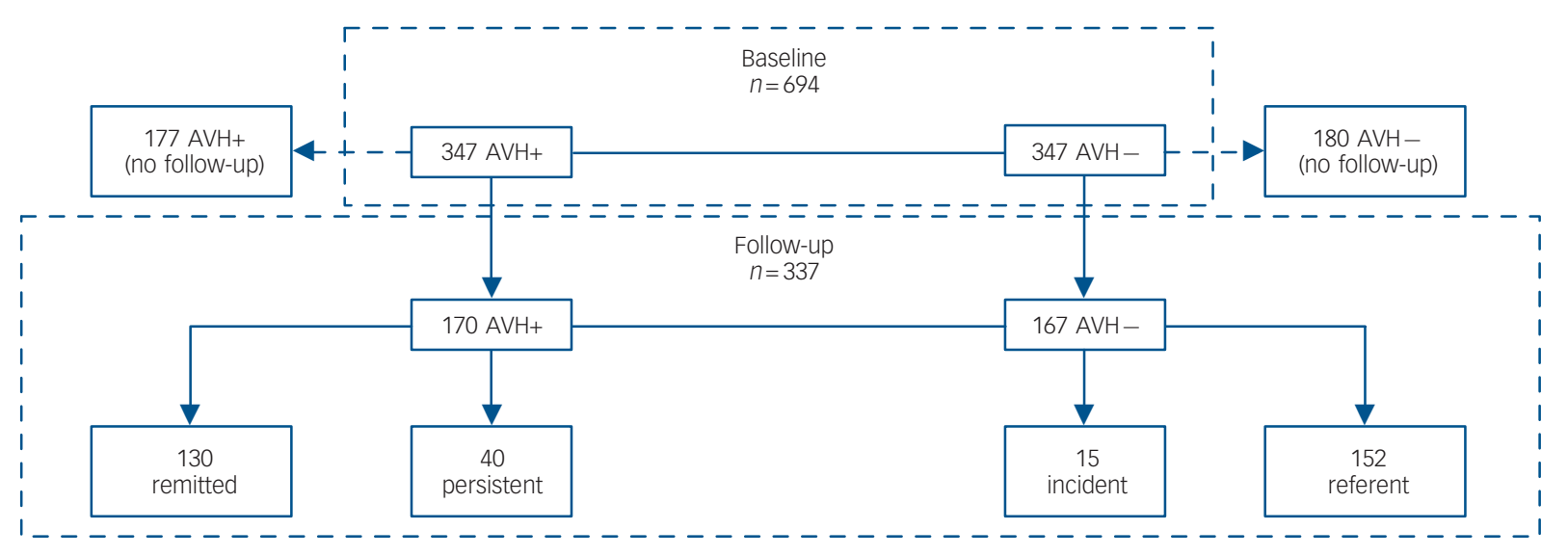

\section{Fig. 1 Flow chart of auditory vocal hallucinations in baseline and follow-up participants.}

\begin{tabular}{|c|c|c|c|c|c|c|}
\hline & $\begin{array}{l}\text { Incident group } \\
\quad(n=15)\end{array}$ & $\begin{array}{l}\text { Persistent group } \\
\qquad(n=40)\end{array}$ & $\begin{array}{l}\text { Remitted group } \\
\qquad(n=130)\end{array}$ & $\begin{array}{l}\text { Referent group } \\
\qquad(n=152)\end{array}$ & Test & P \\
\hline Age, years: mean & 13.2 & 13.1 & 13.1 & 13.2 & $F=0.59$ & ns \\
\hline Female, \% & 60 & 55 & 53 & 54 & $\chi^{2}=0.28$ & ns \\
\hline \multicolumn{7}{|c|}{ Socioeconomic status, \% } \\
\hline Low & 47 & 35 & 32 & 27 & \multirow{3}{*}{$\chi^{2}=5.06$} & \multirow{3}{*}{ ns } \\
\hline Middle & 27 & 40 & 35 & 44 & & \\
\hline High & 27 & 25 & 33 & 29 & & \\
\hline
\end{tabular}


CI $1.29-2.55, P=0.001)$. Odds ratios were highest when only the narrow psychotic symptoms of the 'thought problems' scale (i.e. 'Strange behaviour', 'Strange ideas' and 'Sees things that aren't there') were included (baseline: persistent auditory vocal hallucinations, $\mathrm{OR}=2.40,95 \% \mathrm{CI} 1.15-5.02, P=0.020$; incident auditory vocal hallucinations, $\mathrm{OR}=3.19,95 \%$ CI $1.33-7.62$, $P=0.009$; follow-up: persistent auditory vocal hallucinations, $\mathrm{OR}=2.37,95 \%$ CI $1.10-5.10, P=0.027$; incident auditory vocal hallucinations, $\mathrm{OR}=4.06,95 \% \mathrm{CI} 1.84-8.97, P=0.001$ ). Child Behavior Checklist scores at follow-up were more often in the clinical range for both the persistent and the incident group (Table 3).

\section{Severity of auditory vocal hallucinations}

At baseline, $27 \%$ of children with auditory vocal hallucinations $(n=347)$ reported severe suffering associated with auditory hallucinations, compared with $35 \%$ of children with auditory vocal hallucinations at follow-up $(n=55)$. The proportion of children with severe auditory vocal hallucinations at follow-up was higher in the incident group $(60 \%)$ than in the persistent group $(25 \%)\left(\chi^{2}=5.91, P=0.02\right)$. Children with severe auditory vocal hallucinations (but not children with mild auditory vocal hallucinations), compared with the referent group, were more likely to show problem behaviour (CBCL total score: baseline, $\mathrm{OR}=1.03,95 \%$ CI 1.00-1.05, $P=0.033$; follow-up, $\mathrm{OR}=1.04$, 95\% CI 1.02-1.06, $P<0.001)$. At follow-up, children with severe auditory vocal hallucinations were more likely to score in the clinical range of psychopathology $(\mathrm{OR}=5.45,95 \%$ CI 2.10 $14.16, P<0.001)$. Severity of auditory vocal hallucinations at follow-up was not associated with gender $\left(\chi^{2}=1.09, P=0.58\right)$, SES $\left(\chi^{2}=3.49, P=0.48\right)$ or urbanicity $\left(\chi^{2}=4.23, P=0.38\right)$. Children with severe auditory vocal hallucinations had lower end-of-primary-school test scores than children with mild auditory vocal hallucinations; both groups had lower scores than control children $(F=6.69, P=0.001)$. Children with severe (but not mild) auditory vocal hallucinations were more likely to attend a lower secondary school type than the referent group $(\mathrm{OR}=4.40$, 95\% CI 1.41-13.73, $P=0.01)$.

\section{Risk factors}

Compared with the referent group, children with incident auditory vocal hallucinations more often lived in an urban environment ( $\mathrm{OR}=3.86,95 \% \mathrm{CI} 1.08-13.89, P=0.038$ ). Severity of auditory vocal hallucinations at follow-up was not associated with urbanicity $(\mathrm{OR}=1.02,95 \% \mathrm{CI} 0.23-4.63, P=0.98)$.

Substance use in the sample was negligible; those who ever used cannabis (the only substance used) were in the incident $(n=1)$ and referent $(n=2)$ groups.

\section{Discussion}

\section{Summary of findings}

The majority $(76 \%)$ of the children with auditory vocal hallucinations at age 7 or 8 years no longer heard voices at the age of 12 and 13 years. This is in line with remission rates presented in a recent systematic review, ${ }^{1}$ which reported that approximately $75-90 \%$ of developmental psychotic experiences are transitory and disappear over time. Although the samples in the systematic review all involved older children (from about 11 years of age), the current study suggests that the natural transitory course also applies to children aged 7-8 years at baseline.

The current results suggest that auditory vocal hallucinations may influence variables indexing cognitive ability, as these variables showed strong associations with persistence, but not with incidence. Persistence was associated with greater severity of auditory vocal hallucinations at baseline with some attenuation by the time of follow-up, when incident auditory vocal hallucinations were associated with the greatest level of severity. In line with this was the finding that the incident group displayed a nearly 5 times, and the persistent group an approximately 2.5 times greater risk for CBCL scores in the clinical range at follow-up. Psychotic thought problems were associated with incident and persistent auditory vocal hallucinations, both at baseline and at follow-up. However, the higher odds ratios for psychotic ideation in the incidence group suggest a higher risk in this group. ${ }^{25,26}$ These differences between the incident and the persistent groups suggest that (a) persistent auditory vocal hallucinations may improve over time (the proportion in the severe range dropped from $40 \%$ at baseline to $25 \%$ at follow-up) and (b) the first manifestation of auditory vocal hallucinations in later childhood may be indicative of more severe underlying pathology.

\section{Findings and initial hypotheses}

The course of auditory vocal hallucinations did not always follow an orderly pattern. Thus, children in the persistent group had been hearing voices for an average period of more than 5.7 years, and all had heard voices in the past year. Approximately $50 \%$ of the persistent group heard voices on a more or less regular (continuous) basis, some of them as long as they could remember. However, in other children, the course seemed intermittent during the 5-year follow-up period. Despite the underlying variability in frequency and duration, persistence of hearing voices more often occurred in children with more severe auditory vocal hallucinations at baseline, confirming the first hypothesis.

The second hypothesis was also confirmed. The pattern of results was that baseline psychotic symptoms were associated with incidence and persistence of auditory vocal hallucinations, and had strong associations with CBCL scores in the clinical range. These findings suggest that auditory vocal hallucinations that are associated with a broader range of psychotic symptoms fare worse and give rise to clinical problem behaviour.

At baseline, the rate of auditory vocal hallucinations was higher in rural areas; however, hallucinations were of greater severity and had more functional impact in urban areas. At follow-up, children in the incident group more often lived in an urban environment, but auditory vocal hallucinations severity was not associated with an urban environment, thus only partly confirming the third hypothesis. At baseline, level of urbanicity was established by primary school postal code because children's home addresses were not available. This procedure seemed justified because primary school addresses mostly were in close range of the children's home address. At follow-up, however, home addresses were available, which we used as estimates because the majority of children living in a rural area would then attend secondary schools in an urban environment.

Logistic regression analyses with baseline auditory vocal hallucination characteristics as covariates showed that hearing more than one voice and the attribution of these voices to an external source were the strongest predictors of hearing voices after 5 years. This confirms our fourth hypothesis, in line with findings of Escher and colleagues, ${ }^{4}$ suggesting that children with a secondary attribution of their voices (i.e. the child had indicated explanations of the voices being caused by spirits, ghosts, special gifts, etc.) had a higher risk of voice persistence. Previous work suggests that individuals who continue to hear voices and 

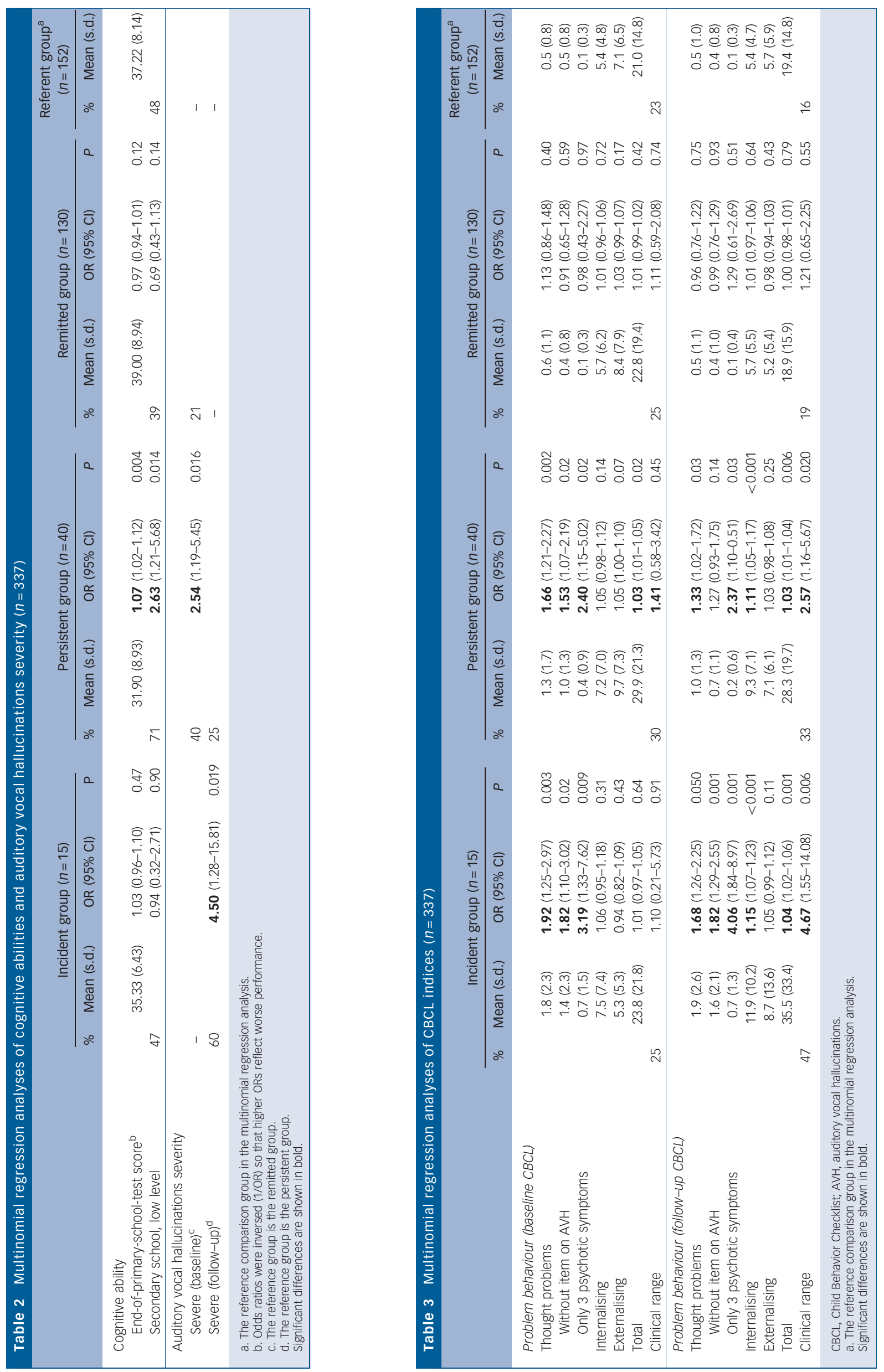
attribute auditory vocal hallucinations to an external source may be more at risk of developing secondary psychotic ideation. ${ }^{27}$

Cognitive ability (indexed by end-of-primary-school test and secondary school level) of children with persistent auditory vocal hallucinations was significantly lower compared with children whose auditory vocal hallucinations were only transitory, and thus may be indicative of risk of transition to psychotic disorder, as suggested by recent studies. ${ }^{17-20,28-30}$ The incident group did not (yet) show alterations in cognitive ability. However, they did have a lower mean score on the end-of-primary-school test (albeit non-significant), which arguably represents a better measure of cognitive ability than secondary school level, given that many other factors intervene in the choice for secondary school type. In addition, the number of children in the incident group was small $(n=15)$. Taken together, these findings provide some support for the fifth hypothesis.

At baseline, associations were tested using prospective data on pre- and perinatal complications, derived from routine infant health service records at baseline assessment; ${ }^{3}$ no clear pattern of association was found. Variables tested at baseline included dichotomous measures on pregnancy, delivery and condition of the child right after birth, as well as continuous variables on early developmental characteristics (in the child's first 12 months of life), summarised in three variables for analysis: fine motor activity, gross motor activity and communication. ${ }^{3}$ Post-hoc examination of these perinatal and developmental variables in relation to the groups at follow-up similarly revealed no significant association for any of the variables (data not shown), indicating that the absence of a clear pattern of association between auditory vocal hallucinations and these variables at baseline was valid.

\section{Clinical implications}

Based on our results, it may be concluded that the majority of auditory hallucinations at baseline are benign and will be transient. If presented in the context of behavioural problems or with other psychotic experiences, it may be advisable for parents to seek help for their child, in order to prevent a poor prognosis. However, parental recognition of the child's auditory hallucinations was low. At baseline, 13\% of the parents indicated that their child may hear 'things', whereas at follow-up, only $25 \%$ of the parents of the persistent group indicated correct recognition of the phenomenon. For clinicians, the findings may be helpful in the sense that in the case of children presenting with somatic complaints, behavioural problems, lower school results or thought problems, the possibility of underlying auditory hallucinations should be considered. On the other hand, when a child reveals the existence of uncomplicated auditory hallucinations, explanations of the mostly transitory nature of the experience may be appropriate.

\section{Limitations}

We could not include all children of the baseline case-control sample. First, $13 \%$ of the parents had already indicated at baseline that they did not want to participate in a follow-up study. Second, despite maximum efforts to include as many children as possible, follow-up participation was not always possible, as parents were sometimes protective towards their children who had just started secondary school and were in a process of adjustment. Third, there is good reason to assume that a number of children did not want to participate a priori. Fourth, some of the non-responding families could not be traced or contacted by telephone. There were, however, no indications that non-response or refusal rates were due to (psychiatric) problems of the children. Baseline participation rates of children with auditory vocal hallucinations and controls were almost equal (50\%). Likewise, the follow-up participation rate of children with severe and mild auditory vocal hallucinations at baseline equals the proportion at baseline $(25 \%$ severe and $75 \%$ mild). These considerations were confirmed by data from the PCR-NN, in which referral and contact rates at group level also showed no differences. Finally, the study design did not permit us to gather any clinical information, including diagnostic assessments, from treatment providers. This would have shed more light on the association between childhood auditory hallucinations and clinical disorders.

Agna A. Bartels-Velthuis, PhD, Gerard van de Willige, MSc, Jack A. Jenner, MD, PhD, University Medical Center Groningen, University of Groningen, Groningen, The Netherlands; Jim van Os, MD, PhD, European Graduate School for Neuroscience, SEARCH, Maastricht University Medical Centre, The Netherlands, and King's College London, King's Health Partners, Department of Psychosis Studies, Institute of Psychiatry, London, UK; Durk Wiersma, PhD, University Medical Center Groningen, University Center for Psychiatry, University of Groningen, Groningen, The Netherlands

Correspondence: Agna A. Bartels-Velthuis, University Medical Center Groningen, University Center for Psychiatry, University of Groningen, PO Box 30.001 (CC72), 9700 RB Groningen, The Netherlands. Email: a.a.bartels@med.umcg.n

First received 10 Sep 2010, final revision 10 Feb 2011, accepted 21 Mar 2011

\section{Funding}

We thank the funding organisations: the 'Stichting tot Steun VCVGZ' (Foundation for Support, Christian Union for Care of Mentally III), the 'Stichting Open Ankh', the 'Bensdorp Fund', Maastricht University Medical Centre and the Rob Giel Research centre of the University of Groningen. The 'Stichting tot Steun VCVGZ', 'Stichting Open Ankh' and 'Bensdorp Fund' had no role in the study design, in the collection, analysis, and interpretation of data, in the writing of the report, or in the decision to submit the paper for publication.

\section{Acknowledgements}

The authors are most grateful to all children and parents who took part in this follow-up study. We thank the interviewers for all their efforts, and we thank our colleagues from the Psychiatric Case Register North-Netherlands for providing data at group level.

\section{References}

1 van Os J, Linscott RJ, Myin-Germeys I, Delespaul P, Krabbendam L. A systematic review and meta-analysis of the psychosis continuum: evidence for a psychosis proneness-persistence-impairment model of psychotic disorder. Psychol Med 2009; 39: 179-95.

2 Kaymaz N, van Os J. Extended psychosis phenotype - yes: single continuum - unlikely. Psychol Med 2010; 40: 1963-6.

3 Bartels-Velthuis AA, Jenner JA, van de Willige G, van Os J, Wiersma D. Prevalence and correlates of auditory vocal hallucinations in middle childhood. Br J Psychiatry 2010; 196: 41-6.

4 Escher S, Romme M, Buiks A, Delespaul $\mathrm{P}$, van Os J. Independent course of childhood auditory hallucinations: a sequential 3-year follow-up study. Br J Psychiatry 2002; 181: s10-8.

5 Askenazy FL, Lestideau K, Meynadier A, Dor E, Myquel M, Lecrubier Y. Auditory hallucinations in pre-pubertal children. Eur Child Adolesc Psychiatry 2007; 16: 411-5.

6 Poulton R, Caspi A, Moffitt TE, Cannon M, Murray R, Harrington H. Children's self-reported psychotic symptoms and adult schizophreniform disorder: a 15-year longitudinal study. Arch Gen Psychiatry 2000; 57: 1053-8.

7 Eminson DM. Medically unexplained symptoms in children and adolescents. Clin Psychol Review 2007; 27: 855-71.

8 Janssens KAM, Rosmalen JGM, Ormel J, Van Oort FVA, Oldehinkel AJ. Anxiety and depression are risk factors rather than consequences of functional somatic symptoms in a general population of adolescents: the TRAILS study. J Child Psychol Psychiatry 2010; 51: 304-12.

9 Welham J, Scott J, Williams G, Najman J, Bor W, O'Callaghan M, et al. Emotional and behavioural antecedents of young adults who screen positive 
for non-affective psychosis: a 21-year birth cohort study. Psychol Med 2009; 39: $625-34$

10 Ott SL, Allen J, Erlenmeyer-Kimling L. The New York High-Risk Project: observations on the rating of early manifestations of schizophrenia. Am J Med Genet 2001; 105: 25-7.

11 Henquet C, Krabbendam L, Spauwen J, Kaplan C, Lieb R, Wittchen HU, et al Prospective cohort study of cannabis use, predisposition for psychosis, and psychotic symptoms in young people. BMJ 2005; 330: 11-4

12 Hides L, Lubman DI, Buckby J, Yuen HP, Cosgrave E, Baker K, et al. The association between early cannabis use and psychotic-like experiences in a community adolescent sample. Schizophr Res 2009; 112: 130-5.

13 Mackie CJ, Castellanos-Ryan N, Conrod PJ. Developmental trajectories of psychotic-like experiences across adolescence: impact of victimization and substance use. Psychol Med 2011; 41: 47-58.

14 Cougnard A, Marcelis M, Myin-Germeys I, De Graaf R, Vollebergh W, Krabbendam $L$, et al. Does normal developmental expression of psychosis combine with environmental risk to cause persistence of psychosis? A psychosis proneness-persistence model. Psychol Med 2007; 37: 513-27.

15 Spauwen J, Krabbendam L, Lieb R, Wittchen HU, van Os J. Evidence that the outcome of developmental expression of psychosis is worse for adolescents growing up in an urban environment. Psychol Med 2006; 36: 407-15.

16 Weiser M, van Os J, Reichenberg A, Rabinowitz J, Nahon D, Kravitz E, et al. Social and cognitive functioning, urbanicity and risk for schizophrenia. Br J Psychiatry 2007; 191: 320-4.

17 Cannon M, Caspi A, Moffitt TE, Harrington H, Taylor A, Murray RM, et al. Evidence for early-childhood, pan-developmental impairment specific to schizophreniform disorder. Results from a longitudinal birth cohort. Arch Gen Psychiatry 2002; 59: 449-56.

18 Horwood J, Salvi G, Thomas K, Duffy L, Gunnell D, Hollis C, et al. IQ and nonclinical psychotic symptoms in 12-year-olds: results from the ALSPAC birth cohort. Br J Psychiatry 2008; 193: 185-91.

19 Jabben N, Van Os J, Janssen I, Versmissen D, Krabbendam L. Cognitive alterations in groups at risk for psychosis: neutral markers of genetic risk or indicators of social disability? Acta Psychiatr Scand 2007; 116: 253-62.

20 Welham J, Isohanni M, Jones P, McGrath J. The antecedents of schizophrenia: a review of birth cohort studies. Schizophr Bull 2009; 35: 603-23.
21 Jenner JA, Van de Willige G. The Auditory Vocal Hallucination Rating Scale (AVHRS). University Medical Center Groningen, University Center for Psychiatry, University of Groningen, 2002.

22 Arseneault L, Cannon M, Witton J, Murray RM. Causal association between cannabis and psychosis: examination of the evidence. Br J Psychiatry 2004; 184: $110-7$.

23 Achenbach TM. Manual for the Child Behaviour Checklist/4-18 [Dutch translation: Verhulst et al, 1996]. Department of Psychiatry, University of Vermont, 1991.

24 Bartels-Velthuis AA, Van de Willige G, Jenner JA, Wiersma D. Assessing Auditory Vocal Hallucinations: The Psychometric Evaluation of the Auditory Vocal Hallucination Rating Scale (AVHRS). University Medical Center Groningen, University Center for Psychiatry, University of Groningen, 2008.

25 Dominguez MD, Wichers M, Lieb R, Wittchen HU, van Os J. Evidence that onset of clinical psychosis is an outcome of progressively more persistent subclinical psychotic experiences: an 8-year cohort study. Schizophr Bull 2010; Oct 28. Epub ahead of print.

26 Smeets F, Lataster T, Dominguez MD, Hommes J, Lieb R, Wittchen HU, et al. Evidence that onset of psychosis in the population reflects early hallucinatory experiences that through environmental risks and affective dysregulation become complicated by delusions. Schizophr Bull 2010; Oct 28. Epub ahead of print.

27 Krabbendam L, Myin-Germeys I, Hanssen M, Bijl RV, De Graaf R, Vollebergh W, et al. Hallucinatory experiences and onset of psychotic disorder: evidence that the risk is mediated by delusion formation. Acta Psychiatr Scand 2004; 110: 264-72.

28 Van Oel CJ, Sitskoorn MM, Cremer MPM, Kahn RS. School performance as a premorbid marker for schizophrenia: a twin study. Schizophr Bull 2002; 28: 401-14.

29 Dominguez MD, Can SM, Lieb R, Wittchen HU, van Os J. Early expression of negative/disorganized symptoms predicting psychotic experiences and subsequent clinical psychosis: a 10-year study. Am J Psychiatry 2010; 167: 1075-82.

30 Weiser M, Werbeloff N, Drukker M, Van Os J, Dohrenwend BP, Yoffe R, et al. Self-reported psychotic symptoms in the community, and risk of later hospitalization for non-affective psychotic disorders. Schizophr Bull 2009; 35 (suppl 1): 74. 